\title{
Detection of active proteasome structures in brain extracts: proteasome features of August rat brain with violations in monoamine metabolism
}

\author{
Pavel A. Erokhovi, ${ }^{1}$, Yulia V. Lyupina ${ }^{1, *}$, Alexandra S. Radchenko ${ }^{1}$, Anna A. \\ Kolacheva $^{2}$, Yulia O. Nikishina ${ }^{2}$ and Natalia P. Sharova ${ }^{1}$ \\ ${ }^{1}$ Laboratory of Biochemistry of Ontogenesis Processes, N.K. Koltsov Institute of Developmental Biology of Russian Academy \\ of Sciences, Moscow, Russia \\ ${ }^{2}$ Laboratory of Neural and Neuroendocrine Regulations, N.K. Koltsov Institute of Developmental Biology of Russian Academy \\ of Sciences, Moscow, Russia \\ *These authors have contributed equally to this work
}

Correspondence to: Natalia P. Sharova, email: npsharova@bk.ru

Keywords: proteasome structure, immune proteasomes, rat brain, violations in monoamine metabolism, native electrophoresis Received: May 26, $2017 \quad$ Accepted: July 23, $2017 \quad$ Published: August 10, 2017

Copyright: Erokhov et al. This is an open-access article distributed under the terms of the Creative Commons Attribution License 3.0 (CC BY 3.0), which permits unrestricted use, distribution, and reproduction in any medium, provided the original author and source are credited.

\section{ABSTRACT}

The aim of this work was to detect changes in proteasome pools of brain parts of August rats with monoamine metabolism violations in comparison with that of control Wistar rats. To reveal active proteasome structures, a method of native electrophoresis for the analysis of crude tissue fractions was developed. By means of this method and following Western blotting, the most pronounced changes in reorganization of proteasome structures were detected in proteasome pool of the brain cortex of August rats. Main findings are the enhanced expression of immune proteasome subtypes containing proteolytic subunit LMP2 and activator PA28a $\beta$ as well as immune proteasome subtypes containing proteolytic subunit LMP7 and activator PA700 and simultaneously decreased expression of subtypes with subunit LMP2 and activator PA700 in the brain cortex of August rats compared to that of Wistar rats. These results were indirectly confirmed by SDS PAGE method followed by Western blotting, which showed the increased quantities of immune subunits and proteasome activators in the brain cortex of August rats compared to that of Wistar rats. Immune proteasomes were revealed by immunohistochemistry in neurons, but not in glial cells of August and Wistar rat cortex. The detected reorganization of proteasome pools is likely to be important for production of special peptides to provide the steady interaction between neurons and adaptation of central nervous system to conditions caused by monoamine metabolism deviations.

\section{INTRODUCTION}

The study of adaptive mechanisms developing in the brain under stress conditions is tightly related to the elucidation of neural plasticity phenomenon. Of great interest is the stress provoked by changes in the content of monoamines especially serotonin. Serotonergic mechanisms play an important role in the regulation of anxiety behavior in mammals [1-3]. At the early stages of the development of central nervous system, synapse formation and elimination depend on serotonin [4]. The inbred line of August rats is characterized by genetically determined disorders in monoamine metabolism and therefore it represents the convenient model for the study of effects of these disorders. In the motor cortex of August rats, the level of biogenic amines and their metabolites is known to be higher than in the midbrain reticular formation in contrast to Wistar rats $[5,6]$. Despite of this imbalance, 
the exchange between the cortex and subcortical structures that manages function of the cerebral cortex, in August rats remains intact [7]. August rats show higher inherent basal activity of stress-limiting systems (NO synthase and heat shock proteins, HSPs) accompanied by the increased resistance to the acute emotional stress in comparison with Wistar rats [8-10].

Other key molecular participators important for adaptation of the brain to deviations in monoamine metabolism remain poorly studied and understood.

It is known that proteasomes, multicatalytic proteases, hydrolyze up to $90 \%$ of proteins in cells and regulate numerous cellular processes. Proteasomes participate in adaptive mechanisms under oxidative stress and stress influenced by the absence of major histocompatibility (MHC) class I molecules [11-14]. In this regard, we focused our attention on proteasome study in the brain of August rats.

Cellular proteasome pool consists of multiple forms differed in the combination of proteolytic subunits and regulators. $20 \mathrm{~S}$ proteasome, or $20 \mathrm{~S}$ core particle, has a barrel-shaped structure that is composed of four rings of seven subunits each, two outer rings of a subunits and two internal rings of $\beta$ subunits [15]. Hydrophobic N-ends of a subunits form barrier preventing the casual penetration of proteins into the proteolytic chamber. Proteolytic activities are associated with the internal $\beta$ rings. Catalytic subunits $\beta 1, \beta 2$, and $\beta 5$ demonstrate caspase-like (CL) (also termed peptidyl-glutamyl peptide-hydrolyzing), trypsin-like, and chymotrypsin-like (ChTL) activities respectively. Mammals have special proteasome forms, immune proteasomes. They contain proteolytic subunits LMP7( $\beta 5 \mathrm{i})$, LMP2( $\beta 1$ i), and MECL1( $\beta 2 \mathrm{i})$ instead of subunits $\beta 5, \beta 1$, and $\beta 2$ of constitutive proteasomes. Replacement of constitutive subunits by immune ones takes place during new proteasome assembling. Subunits LMP2 and MECL1 may be integrated together and independently from LMP7. Although incorporation of subunit LMP7 is facilitated by subunits LMP2 and MECL1, this process can also take place without them $[16,17]$. Both immune and constitutive forms are capable of hydrolyzing the foreign (mutant or viral) and own cellular proteins [18]. The immune proteasomes, like constitutive ones, display ChTL, CL and trypsin-like activities. At the same time, the immune proteasomes have the altered cleavage site preference due to the change in the conformation of substrate binding pockets that affects the structure of the peptides produced [19]. On the whole, in the immune proteasomes ChTL and trypsinlike activities are higher and CL activity is lower than in the constitutive proteasomes. It allows the immune proteasomes to generate antigen epitopes for MHC class I molecules or other special peptides in a large quantity.

$20 \mathrm{~S}$ proteasome is the smallest functional structure of $750 \mathrm{kDa}$ able to cleave a number of substrates including proteins with damaged conformation under oxidative stress and several viral proteins $[11,12,20,21]$. The specificity of ubiquitinated protein hydrolysis is ensured by $26 \mathrm{~S}$ proteasome of approximately $2.5 \mathrm{MDa}$ consisting of 20S core particle and PA700 (19S) activator [22, 23]. After recognition and binding of ubiquitinated proteins, PA700 activator carries out their deubiquitination and ATPdependent unfolding. Multifunctionality of this activator is provided by 20-21 subunits that organize its structure. On the whole, $26 \mathrm{~S}$ proteasomes secure the protein rotation in cells and elimination of damaged proteins.

The specificity of proteasome functioning depends not only on PA700 activator and immune subunits, but also on activators PA28 (11S) and PA200, flanking proteasome $20 \mathrm{~S}$ core particle. Cytoplasmic activator PA28a $\beta$, containing $3 a$ and $4 \beta$ (or $4 a$ and $3 \beta$ ) subunits of $28 \mathrm{kDa}$ each, stimulates hydrolysis of unstructured proteins and polypeptides of middle and small length. Proteasomes with PA700 and PA28 activators may produce special peptides for MHC class I molecules even in the absence of proteolytic immune subunits [18, 24, 25]. Activator PA200, the protein of $200 \mathrm{kDa}$, regulates proteasome proteolytic activity by opening of a canal for not long substrates. It is also possible that activator PA200 facilitates the release of hydrolysis products [26].

Different sets of multiple proteasome forms provide the specific functions of each tissue and organ. The aim of this work was to detect changes in proteasome pools of the brain parts of August rats with monoamine metabolism violations in comparison with that of control Wistar rats. This aim was achieved with the use of native electrophoresis method developed for the analysis of proteasomes in crude tissue fractions.

\section{RESULTS AND DISCUSSION}

\section{Characteristic of monoamine metabolism and HSP70 expression in the brain parts of August rats}

First of all, we confirmed the presence of changes in the content of serotonin and dopamine in the brain parts of August rats taken in the experiment, in comparison with that of Wistar rats. The content of both monoamines in the cortex and striatum in August rats was higher than in Wistar rats (Table 1). The lower ratio of the content of homovanillic acid (dopamine metabolite) and dopamine, as well as 5-hydroxyindoleacetic acid (serotonin metabolite) and serotonin in the cortex and striatum of August rats indicates the less rate of dopamine and serotonin metabolizing in these brain parts of August rats compared to Wistar rats (Table 1). In the brainstem of August rats, the level of dopamine, but not serotonin, was higher in relation to that of Wistar rats due to the less intensity of metabolizing processes detected by the ratio of the quantity of dopamine metabolites, 3,4-dihydroxyphenylacetic acid and homovanillic acid, to dopamine (Table 1). 
Table 1: Content of serotonin, dopamine and their major metabolites in brain parts of Wistar and August rats

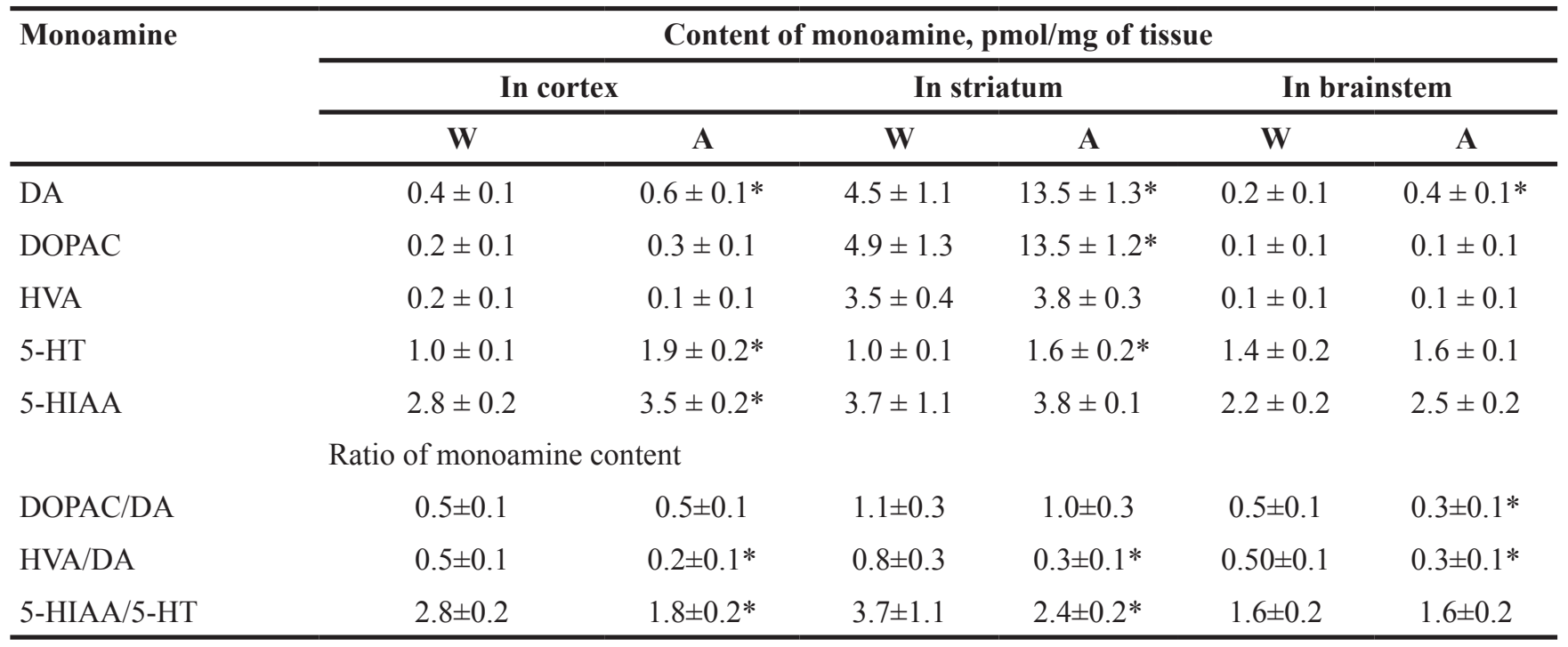

A, August rats; W, Wistar rats; DA, dopamine; DOPAC, 3,4-dihydroxyphenylacetic acid; HVA, homovanillic acid; 5HT (5-hydroxytryptamine), serotonin; 5-HIAA, 5-hydroxyindoleacetic acid.

Standard deviation from the average magnitude is shown. * Reliable difference from corresponding control magnitude $(p<$ $0.05, \mathrm{n}=6)$.
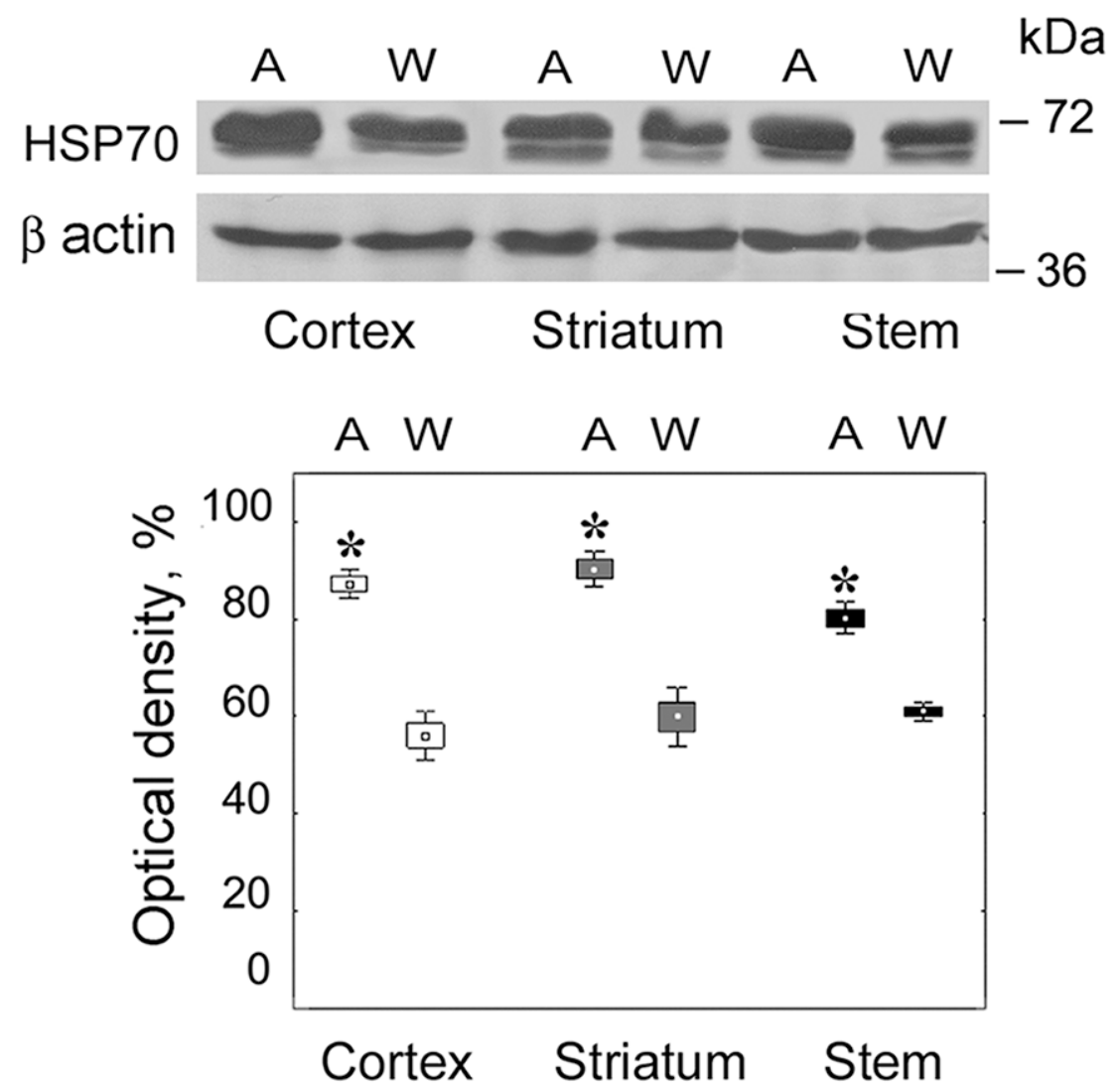

Figure 1: Content of HSP70 in brain parts of August and Wistar rats. On the top, Western blots of HSP70 with the use of corresponding antibodies. Molecular mass of standard protein markers is shown. On the bottom, relative subunit quantities as percentage from the maximum magnitude normalized to $\beta$ actin level and presented as means and SEM. *Reliable difference from control magnitude $(p=0.02, \mathrm{n}=8)$. A, August rats; W, Wistar rats. 
We revealed the enhanced level of stress-limiting HSP70 protein in all studied brain areas of August rats in comparison with Wistar rats $(\mathrm{H}(1,32)=5.23 ; p=0.02)$ (Figure 1). This result indicated the stress conditions in the brain of August rats.

Thus, the brain of August rats taken in the experiment was characterized by the presence of the stress associated with monoamine metabolism deviations.

\section{Expression patterns of proteasome subunits in the brain parts of August rats}

To detect changes in the expression of proteasome subunits, the standard approach with the use of SDS PAGE followed by Western blotting was applied. Only the protein content of proteasome subunits (not mRNA content) was determined, because mRNA levels were not well correlated with the amounts of proteasome proteins, suggesting a specific regulation of proteasome subunit synthesis [27]. The relative content of proteasome proteolytic constitutive subunits $\beta 1, \beta 5$ and immune subunits LMP2, LMP7 was evaluated with utilizing the antibodies to these subunits. Because of the joint incorporation of LMP2 and MECL1 subunits into proteasome structure [16], it was enough to analyze LMP2 expression patterns for the evaluation of changes in the expression of both subunits. The level of activator PA200 was detected with the use of antibodies to this protein. The expression patterns of activators PA700 and PA28a $\beta$ were studied with the application of antibodies to subunits Rpt6 and PA28a included in the structures of these activators respectively. The total proteasome pool was studied by means of combined antibodies to subunits a1,2,3,5,6,7 incorporated in all proteasome forms.

Proteasome subunit levels were dependent on the brain structure and rat strain $(\mathrm{H}(1,18)=0.235 ; p=0.015)$. The most marked changes in the proteasome pool were revealed in the brain cortex of August rats compared to that of control Wistar rats. In this brain part, the expression of immune proteolytic subunits LMP7 $(\mathrm{H}(1,8)=5.46 ; p=$ $0.01)$ and LMP2 $(\mathrm{H}(1,8)=5.43 ; p=0.02)$ was three times higher (Figure $2 \mathrm{~A}$ and $2 \mathrm{~B})$. The expression of activators PA700 $(\mathrm{H}(1,8)=3.88 ; p=0.04), \mathrm{PA} 28 \mathrm{a} \beta(\mathrm{H}(1,8)=5.36$; $p=0.03)$ and PA200 $(\mathrm{H}(1,8)=5.46 ; p=0.01)$ was also enhanced but to the less degree, up to $40 \%$.

In the striatum of August rats, the content of LMP7 $(\mathrm{H}(1,8)=5.33 ; p=0.02)$ and LMP2 $(\mathrm{H}(1,8)=5.36 ; p=$ 0.03 ) subunits was two times higher (Figure 2C and 2D). The expression of proteasome activators showed the tendency to increase, but reliable differences were not detected.

In the stem, only the expression of LMP7 subunit $(\mathrm{H}(1,8)=3.85 ; p=0.04)$ was higher (by 50\%) in comparison with control (Figure $2 \mathrm{E}$ and $2 \mathrm{~F}$ ).

The level of the total proteasome pool was increased by $20 \%$ and $25 \%$ in the striatum and cortex of August rats respectively, in the stem it was identical in August and Wistar rats (Figure $2 \mathrm{G}$ and $2 \mathrm{H}$ ). At the same time, there was no expected reliable decrease, except of the tendency to decrease, in the content of constitutive subunits $\beta 1$ and $\beta 5$ replaced by immune subunits LMP2 and LMP7 (Figure 2A-2F). This apparent contradiction may be explained by the small portion of the immune subunits in comparison with the content of constitutive ones in the total proteasome pools. So, the essential increase of the quantity of the immune subunits is reflected in unessential and unreliable decrease of the level of the constitutive subunits.

Note that the immune proteasomes are known to be present mainly in the organs of the immune system. So, we compared the quantities of LMP7 and LMP2 subunits in extracts of rat brain cortex and thymus. At the same time, thymus extract may serve as a positive control of the immune subunit presence. In the brain cortex, much lesser quantities of the immune subunits were detected (Figure 3 ). Normalized to $\beta$ actin content, the quantities of LMP7 and LMP2 subunits were 8 and 15 times less in the brain cortex than in thymus. Normalized to the total proteasome pool, the content of these subunits was respectively 4 and 7 times less in the brain cortex than in thymus. In this regard, the appropriate choice of tissue extract volume, that would depend on the peculiarities of the proteasome pool, is important for the immune proteasome investigation. Besides, extract of insect Sf9 cells prepared as earlier described [28] served as a negative control of the immune subunit presence.

The data obtained showed the interesting information about the brain proteasome subunits of August and Wistar rats, but they did not allow us to realize the characteristics of the individual proteasome subtypes in the brain of these rats. We took into account the possibility that not all proteasome subunits detected in this experiment were incorporated in proteasome structure. So, preliminarily we had to develop native electrophoresis method appropriate for the analysis of proteasomes in crude tissue fractions. The investigation of impure tissue extracts allows us to discover the multiplicity of proteasome structures more correctly compared to purified samples whose preparation is often accompanied by the loss of some proteasome forms.

\section{Detection of special conditions for native electrophoresis}

The method was modified on the basis of published protocol for native electrophoresis [28]. Conditions for native electrophoresis were detected with the use of rat liver extract as a fraction most contaminated by microsomes. Nucleic acids and microsomes in probes impede the entry of high molecular mass proteins into gel and constitute the main problem of crude extract electrophoresis at standard initial voltage of $140 \mathrm{~V}$. Under these conditions, 
proteasomes nonspecifically combined with microsomes and / or nucleic acids are distributed throughout the gel beginning from the start position (Figure 4). We solved this problem by low initial voltage of $60 \mathrm{~V}$ applied for $14 \mathrm{~h}$ (Figure 4).

Note that proteasome activity in the gel looked as blurred zones formed by the diffusion of short reaction products.

\section{Expression patterns of proteasome subtypes in the brain cortex of August rats}

By means of method of native PAGE followed by Western blotting, six active proteasome forms differed in the combination of activators were revealed in the brain parts of August and Wistar rats (Figure 5). We conditionally marked two zones, I and II, in the gel. In zone I, active
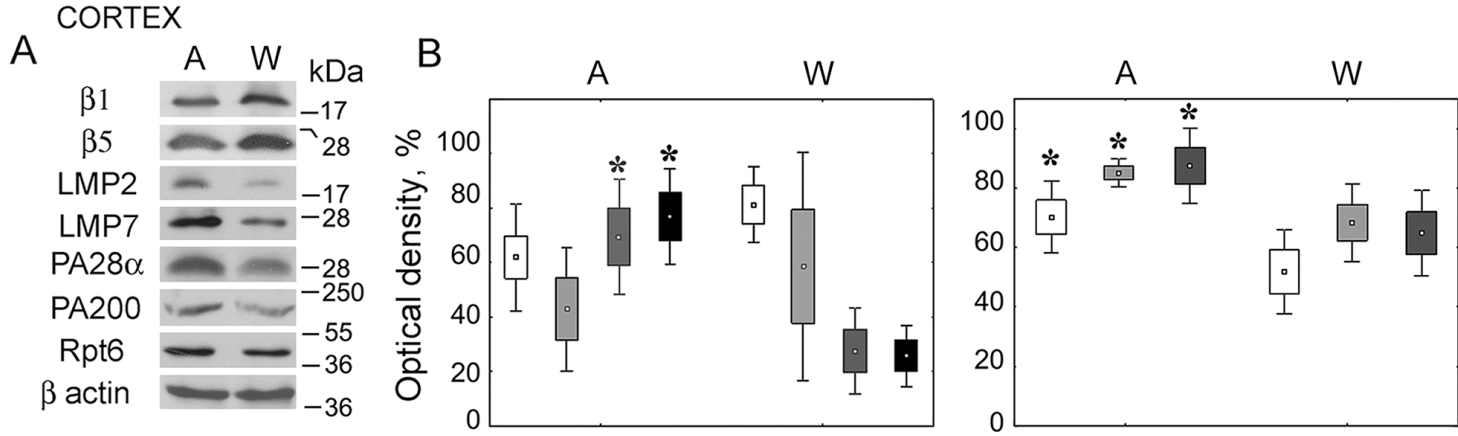

C STRIATUM
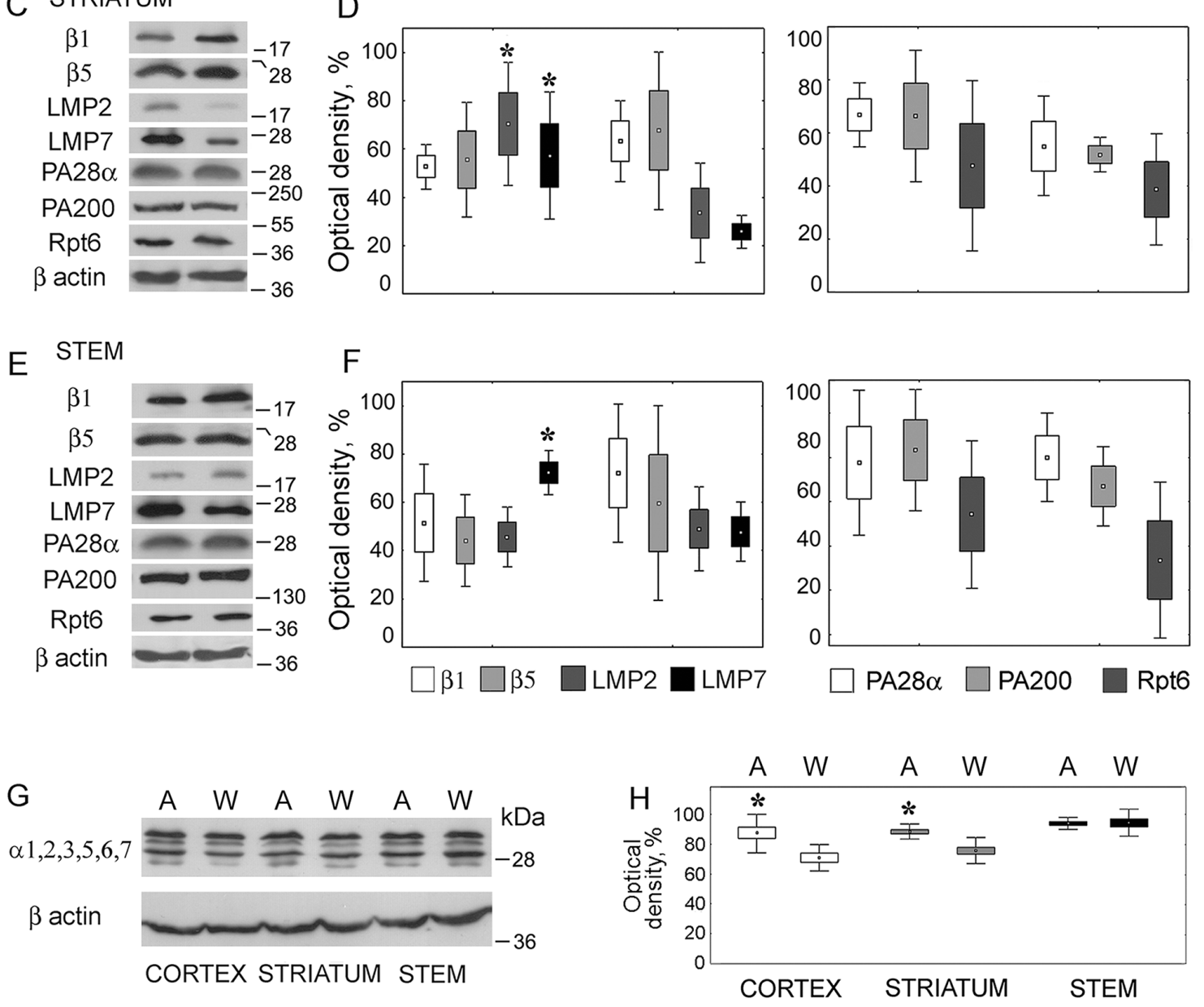

Figure 2: Expression of proteasome and activator subunits in brain parts of August and Wistar rats. (A, C, E, G) Western blots of proteasome and activator subunits with the use of corresponding antibodies. Molecular mass of standard protein markers is shown. $(\mathbf{B}, \mathbf{D}, \mathbf{F}, \mathbf{H})$ The relative subunit quantities as percentage from the maximum magnitude normalized to $\beta$ actin level and presented as means and SEM. *Reliable difference from control magnitude $(p<0.05, \mathrm{n}=8)$. A, August rats; W, Wistar rats. 
proteasome structures of high molecular mass due to the presence of activator PA700 are detected. In fact, they represent different forms of 26S proteasomes: PA700-20S, PA700-20S-PA28aß, PA700-20S-PA200. Zone II contains active proteasome structures with lower molecular mass: PA28a $\beta-20$ S, PA28a $\beta-20 S-P A 28 a \beta$ and PA28a $\beta-20$ SPA200.

Earlier, under other electrophoresis conditions three proteasome forms were detected in the rat cortex: two forms coupled with PA700 activator, and a form free of PA700 activator [29]. It is very probable that short electrophoresis time of 4.5-6.5 h, applied in this work, was insufficient for the high resolution of proteasome structures. In other study, the use of electrophoresis time of $41 \mathrm{~h}$ for the division of partially purified proteasome pool of rabbit reticulocytes into separate proteasome forms allowed the authors to achieve high resolution [30].

Of great interest is the fact of the absence of free $20 \mathrm{~S}$ proteasomes in the brain. This is discovered firstly by the location of $670 \mathrm{kDa}$ marker (Figure 5A, 5D and $5 \mathrm{~F}$ ), secondly by the comparison of electrophoretic lanes with brain samples and standard sample of liver extract (Figure 5A) where 20S proteasomes have been detected earlier [31]. Besides, there is no proteasome band (labeled by $\mathrm{mAb}$ to $\mathrm{a} 1,2,3,5,6,7$ subunits) in the gel free of any proteasome activator (Figure $5 \mathrm{~B}$ ). Taking into account the major role of $20 \mathrm{~S}$ proteasomes as the remover of damaged proteins $[11,12]$, it is consistent to conclude that the brain possesses effective stress-limiting systems which allow it to function without this proteasome form.
Also, intriguing results concerning the expression of proteasome immune subtypes were obtained. Although SDS PAGE and the following Western blotting showed approximately equal increase of LMP2 and LMP7 subunit content in the brain cortex of August rats compared to that of Wistar rats (Figure 2A and 2B), native PAGE detected different changes in the expression of various subtypes containing these subunits.

The most pronounced changes in the expression patterns belong to the immune subtypes containing LMP2 subunit. In the brain cortex of Wistar rats, the major quantity of LMP2 subunit is revealed in the proteasome structures bound to activator PA700, while in August rat cortex LMP2 subunit is mainly detected in proteasome structures bound to activator PA28a $\beta$ (Figure 5B and $5 C)$. Since subunit LMP2 may be included in proteasome together with LMP7 or $\beta 5$ subunit [16], it was important to compare the expression of all these subunits. The distribution of $\beta 5$ subunit, but not LMP7 subunit, in gel zones I and II had similarities with that of LMP2 subunit (Figure 5B and 5C, Table 2). This indicates the decrease of the expression of subtypes $\beta 5$-LMP2 bound to activator PA700 and increase of the expression of subtypes 35 LMP2 bound to activator PA28a $\beta$ in August rat cortex compared to that of Wistar rats. The content of activator PA28a $\beta$ was also increased in gel zone II corresponding to the sample of August rat cortex relatively that of Wistar rat cortex (Table 2). Detected changes were reflected in the proteasome pools, labeled by $\mathrm{mAb}$ to $\mathrm{a} 1,2,3,5,6,7$ subunits, in gel zones I and II (Figure 5B and 5C, Table 2). In gel zone I, the quantity of subunits LMP7, $\beta 1$ and Rpt6

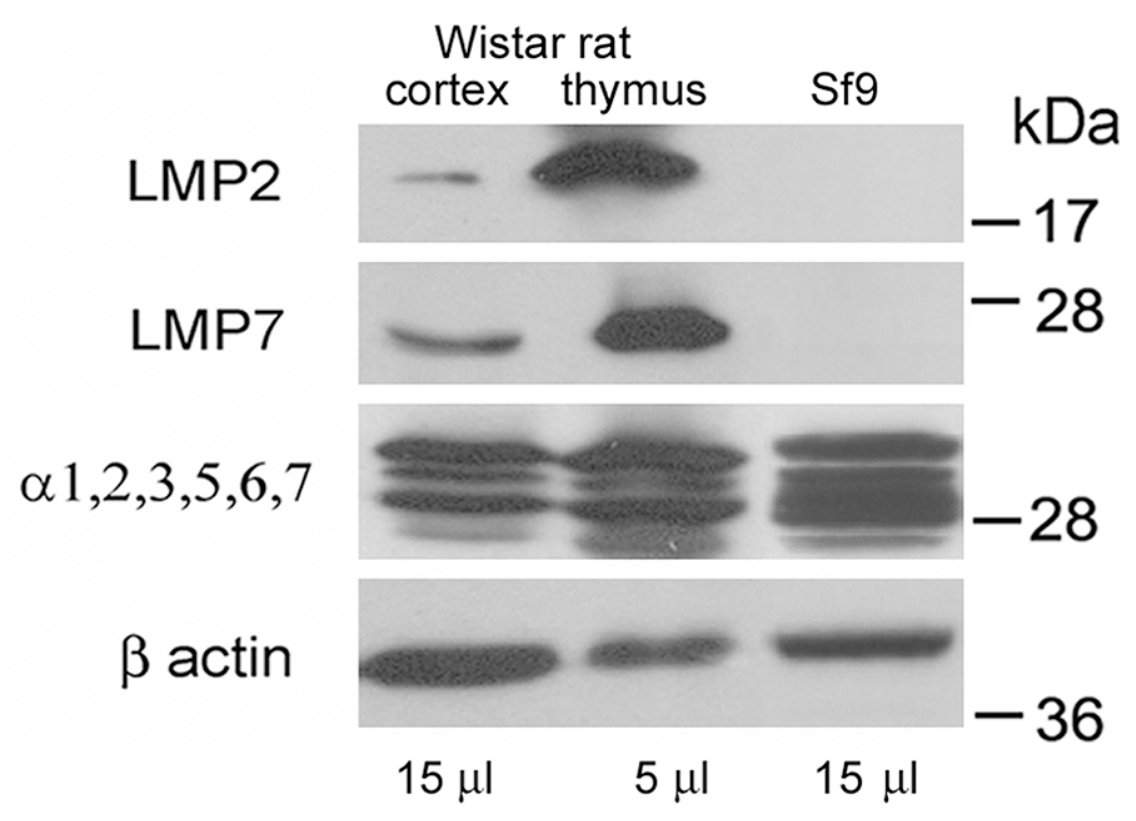

Figure 3: Western blots of proteasome subunits of rat organs and insect cells. Extract of Wistar rat thymus was used as a positive control; extract of Spodoptera frugiperda Sf9 cells was used as a negative control. Molecular mass of standard protein markers is shown. 
is higher for the brain cortex of August rats (Figure 5B, Table 2) indicating the increase of the expression of proteasome subtype LMP7- $\beta 1$ bound to PA700 activator. In gel zone II, the quantity of subunit LMP7 is the same for the brain cortex of August and Wistar rats (Figure $5 \mathrm{~B}$, Table 2). It shows the equal content of proteasome structures with subunit LMP7 and activator PA28a $\beta$.

The analysis of the expression patterns of constitutive proteolytic subunits $\beta 5$ and $\beta 1$ required our due attention. If we take into consideration the presence of these subunits only in immune subtypes $\beta 5$-LMP 2 and LMP7- $\beta 1$, some results would be unclear. First, in gel zone I with the sample of August rat cortex the content of $\beta 5$ subunit is decreased to a greater extent than the content of LMP2 subunit compared to that of Wistar rat cortex (Table 2). Second, in the same sample the increase of $\beta 1$ subunit content is unreliable at $p<0.05$ in contrast to LMP7 subunit content (Table 2). Third, in gel zone II with the sample of August rat cortex the content of $\beta 5$ subunit is increased to a smaller extent than the content of LMP2 subunit compared to that of Wistar rat cortex (Table 2). Fourth, in the same zone the content of $\beta 1$ subunit is reliably decreased in contrast to LMP7 subunit content (Table 2). Taken together, all these facts indicate the presence of the constitutive structures containing $\beta 5$ and $\beta 1$ subunits in both gel zones. The quantity of these structures is diminished in the brain cortex of August rats compared to that of Wistar rats.

The content of activator PA200 is enhanced in both gel zones corresponding to the brain cortex sample of August rats compared to that of Wistar rats (Figure 5B, Table 2). Note that proteasome structures containing

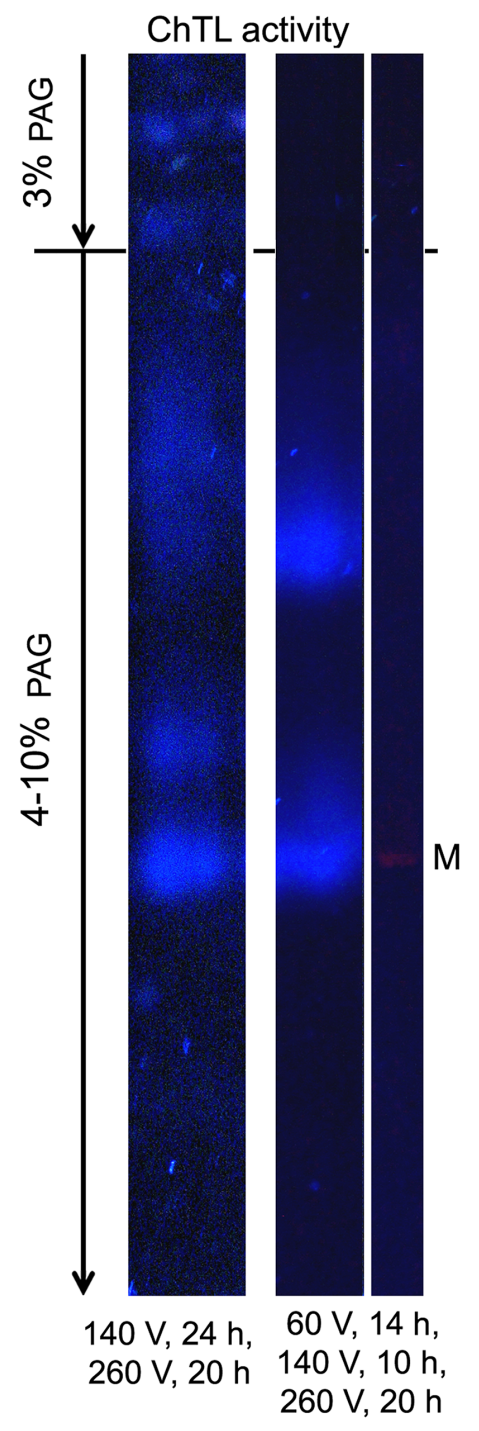

Figure 4: Proteasome ChTL activity of liver extract in the gel after native electrophoresis under different voltage conditions. Thyroglobulin $(670 \mathrm{kDa})$, labeled by the dye Cy-3.5, was applied as a marker $(\mathrm{M})$ of molecular mass. The distribution of activity in the gel was identical in all experiments $(n=6)$. 
activator PA200 or PA28a $\beta$ are revealed in the neighboring locations in the gel. This fact arises from the similar molecular mass of these activators, $200 \mathrm{kDa}$ for activator PA200 and $196 \mathrm{kDa}(28 \mathrm{kDa} \times 7)$ for activator PA28aß.

It is interesting that the expression of the immune subunits in August rat cortex increases more significantly than the expression of the proteasome activators that was detected by both methods (Figure 2A and 2B, Table 2). Such blurred dynamics of the activator expression may be connected with two opposite processes in the cortex of August rats compared to Wistar rats: the increase of the expression of the immune proteasome subtypes bound

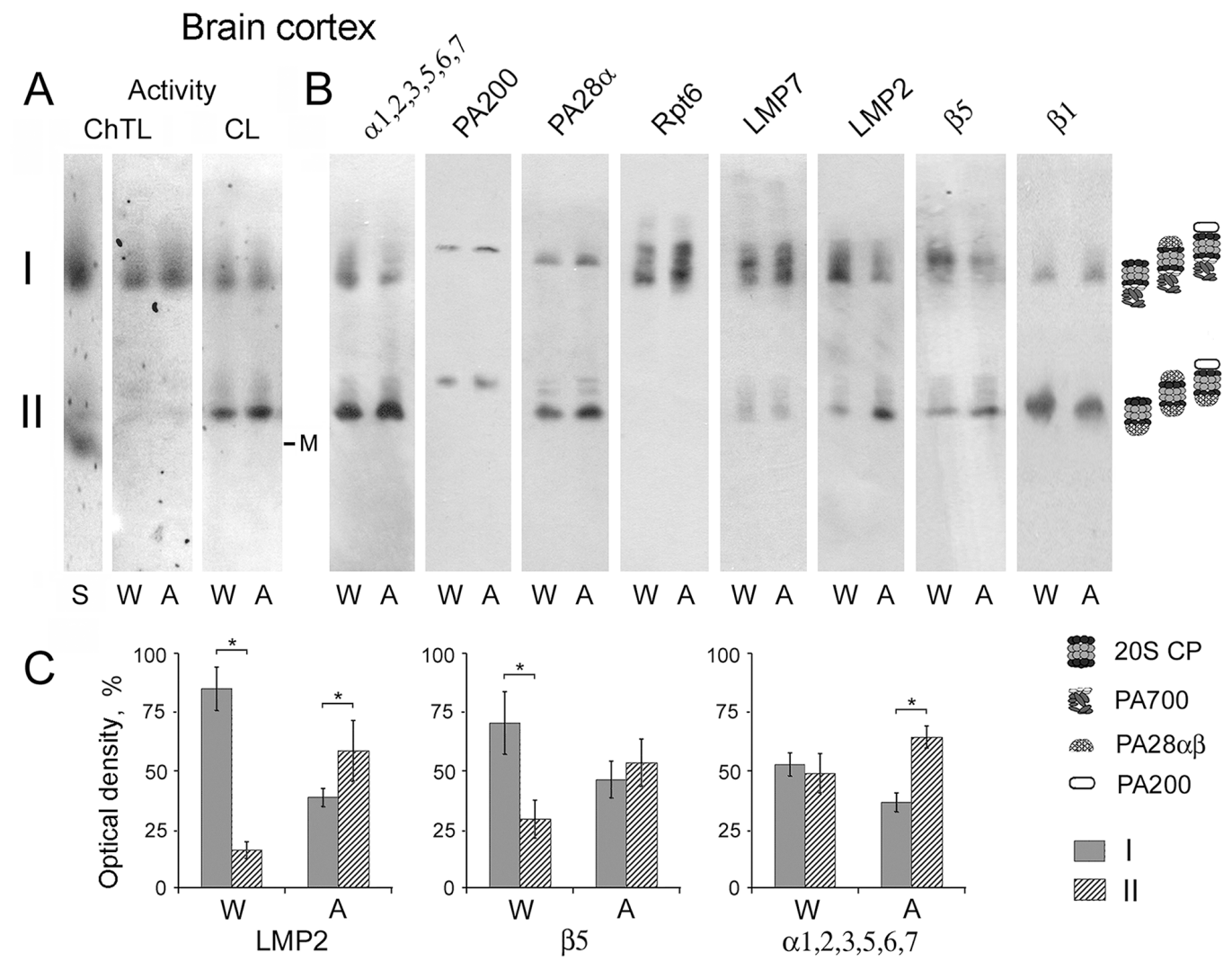

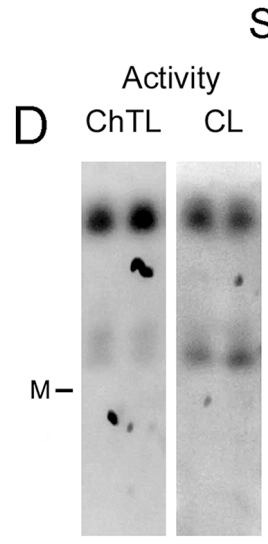

W A W A
Striatum

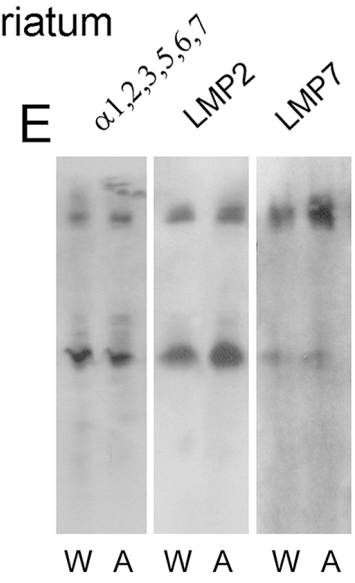

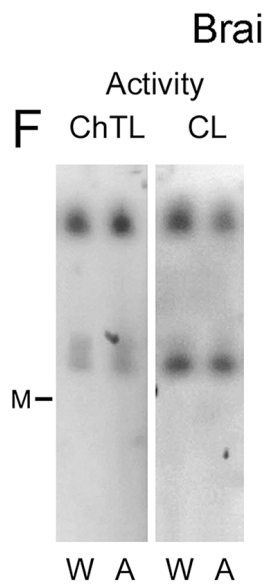

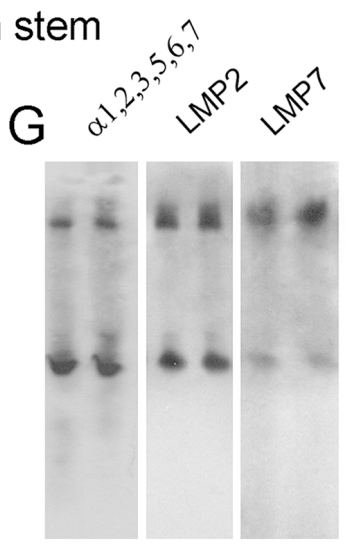

Figure 5: Electrophoresis of brain extracts of Wistar and August rats in native conditions. (A, D, F) Proteasome ChTL and CL activities of the brain cortex, striatum and stem in the gel. Thyroglobulin $(670 \mathrm{kDa})$, labeled by the dye Cy-3.5, was used as a marker (M) of molecular mass. Extract of Wistar rat liver was used as a standard (S). (B, E, G) Western blots of proteasome and activator subunits with the use of corresponding antibodies. (C) The relative subunit quantities in discrete zones I and II as percentage from total quantity in zone I + zone II after electrophoresis of the brain cortex proteins. Error bars, SEM. ${ }^{*}$ Data reliably differ from $\operatorname{control}$ at $p<0.05, \mathrm{n}=6 . \mathrm{A}$, August rats; W, Wistar rats. 
Table 2: Relative quantity of proteasome and activator subunits in brain cortex of August rats compared to that of Wistar rats

\begin{tabular}{lcc}
\hline Proteasome subunit & \multicolumn{2}{c}{ Relative quantity, $\%$} \\
\cline { 2 - 3 } & In gel zone I & In gel zone II \\
\hline LMP2 & $59 \pm 12^{*}$ & $213 \pm 63^{*}$ \\
LMP7 & $180 \pm 32^{*}$ & $101 \pm 10$ \\
$\beta 1$ & $140 \pm 41$ & $60 \pm 25^{*}$ \\
$\beta 5$ & $39 \pm 16^{*}$ & $143 \pm 20^{*}$ \\
PA28a & $126 \pm 24$ & $137 \pm 16^{*}$ \\
Rpt6 & $138 \pm 14^{*}$ & - \\
PA200 & $143 \pm 23^{*}$ & $153 \pm 27^{*}$ \\
a1,2,3,5,6,7 & $62 \pm 10^{*}$ & $146 \pm 23^{*}$ \\
\hline
\end{tabular}

Data are presented as means and SEM. *Reliable difference from control magnitude at $p<0.05, \mathrm{n}=6$.

to the activators and decrease of the expression of the constitutive proteasomes bound to these activators.

Thus, the data obtained demonstrate at least three proteasome subtypes differed in the combination of proteolytic subunits in the brain cortex of August and Wistar rats: LMP7- $\beta 1, \beta 5-\mathrm{LMP} 2$ and $\beta 5-\beta 1$. All these subtypes are bound to activator PA700 or activator PA28a $\beta$. Detected changes in the expression of proteasome subtype groups in the cortex of August rats in comparison with that of Wistar rats are represented in Figure 6.
What do these changes mean? Based on the functions of the indicated subtypes, one may conclude that they provide the production of special biologically active peptides important for the development of adaptive processes under the stress evoked by monoamine metabolism deviations. Perhaps, the process of the peptide production is carried out in two steps. At first, proteasomes containing subunits LMP7, $\beta 1$ and activator PA700 produce polypeptides of middle length from full size proteins in ubiquitin-dependent manner. Then
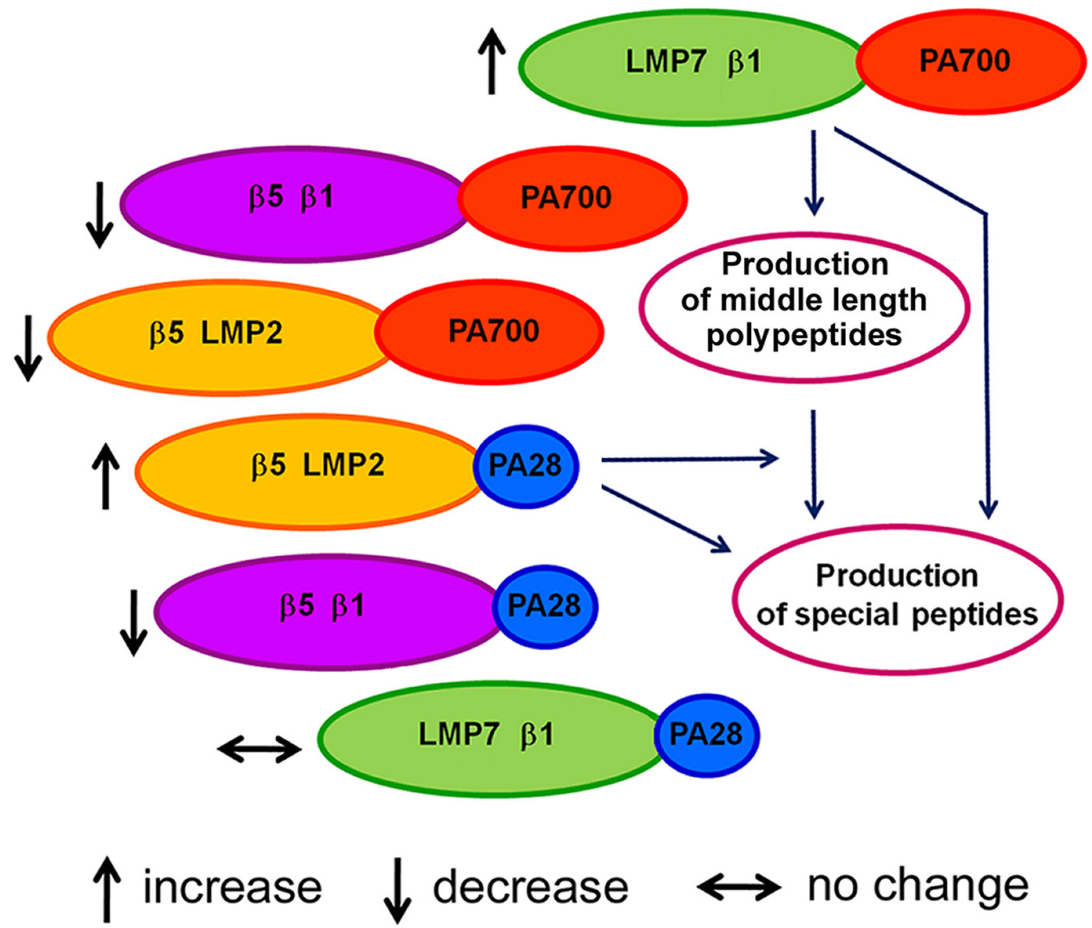

Figure 6: Scheme of changes in proteasome pool of August rat cortex compared to Wistar rat cortex. 
the utilization of these polypeptides and formation of biologically active peptides from them are carried out by proteasomes containing subunits $\beta 5, \mathrm{LMP} 2$ and activator PA28a $\beta$. It is also possible that each of these immune proteasome subtypes is capable of individual production of special peptides. PA200 activator is likely to assist this process by the rapid release of hydrolysis products from the proteolytic cavity. Although the process of production of biologically active peptides in August rat cortex requires the separate investigation, it is already possible to assert the participation of detected immune proteasome subtypes in it.

\section{Expression patterns of proteasome subtypes in the brain stem and striatum of August rats}

In spite of the changes of the proteasome pools in the striatum and stem of August rats in relation to Wistar rats are not as significant as in the cortex according to SDS PAGE and Western blotting, we analyzed the content of the immune subunits in these brain parts by native PAGE. It was shown that in August rat striatum, the content of LMP7 (but not LMP2) subunit was validly higher (by $80 \%$ at $p<0.05)$ in the proteasome structures bound to activator PA700, while the content of LMP2 (but not LMP7) subunit was validly increased (by $60 \%$ at $p<0.05$ ) in the proteasome structures bound to activator PA28a $\beta$ (Figure $5 \mathrm{E})$. In August rat stem, the reliably enhanced content (by $40 \%$ at $p<0.05$ ) of LMP7 (but not LMP2) subunit in the proteasome structures bound to activator PA700 was detected (Figure 5G). This means that the enhanced expression concerns proteasome subtype containing proteolytic subunits LMP7 and $\beta 1$ as well as subtype with proteolytic subunits $\beta 5$ and LMP2 (but not subtype with subunits LMP7 and LMP2).

Although the quantities of the proteasome activators of the striatum and stem in August and Wistar rats do not reliably differ (Figure $2 \mathrm{C}-2 \mathrm{~F}$ ), it is possible that the increased content of proteasome immune subtypes bound to the activators is connected with the decreased content of the constitutive proteasomes bound to these activators.

Thus, despite the differences in the proteasome expression patterns in all studied brain areas of August rats, the increased expression is concerned with the same subtypes of the immune proteasomes, $\beta 5$-LMP2-PA28a $\beta$ and / or LMP7- $\beta 1-P A 700$, indicating their important role in the maintaining of the neural plasticity.

Note that one more study of proteasome structure shows the existence of LMP2 subunit only in the combination with LMP7 subunit in proteasomes of some human organs [32]. Our results indicate the existence of LMP7 and LMP2 subunits in the different proteasome forms rather than in the common proteasome form in rat brain parts and they are in agreement with the study of M. Groettrup and coauthors [16].

\section{Proteasome activities in the brain parts of August and Wistar rats}

It is interesting that ChTL and CL activities in the brain cortex and striatum of August rats were slightly decreased or showed the tendency to decrease compared to that of Wistar rats (Figure 7). Only brain stem of August rats had the enhanced ChTL activity that correlated with the increased expression of LMP7 subunit displaying this activity. At the same time, in this brain area of August rats, CL activity was decreased by $20 \%$ in comparison with that of Wistar rats. On the whole, the changes in proteasome activities of the brain stem may be explained by the change of LMP7 subunit expression.

With regard to the cortex and striatum of August rats, the complex changes in the expression of the proteasome subunits and activators, compared to that of Wistar rats, did not significantly influence proteasome

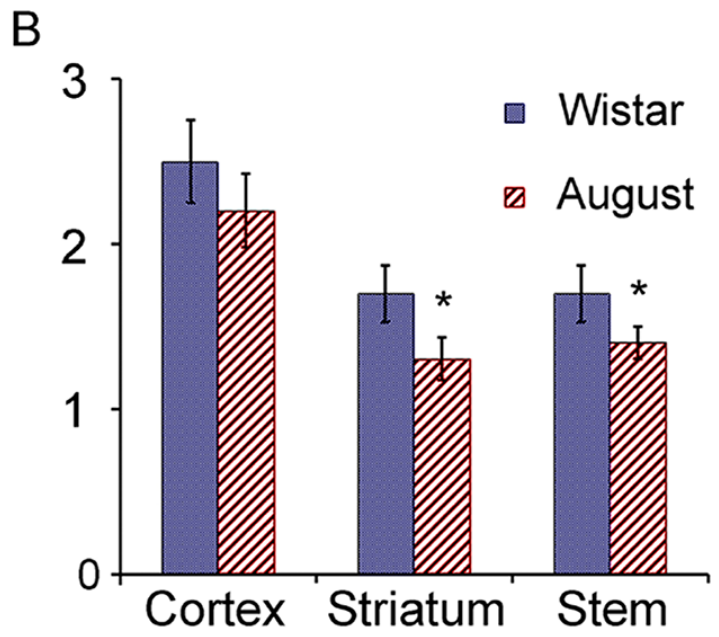

Figure 7: Proteasome ChTL and CL activities in brain parts of Wistar and August rats. (A) ChTL activity, (B) CL activity. Error bars, SEM. *Reliable difference from corresponding activity of Wistar rats $(p<0.05, \mathrm{n}=8)$. 
activities measured with the use of short substratesoligopeptides. Most likely, the changes in proteasome pools in these brain areas of August rats are directed to the alteration of the specificity of hydrolysis of full-size and middle-size proteins with the maintenance of the ability to total cleavage of short substrates.

Although proteasome activities in the gel were detected for revealing the active proteasome zones, some additional interesting information was obtained. Proteasomes in gel zone I (26S proteasomes) possessed significantly higher ChTL activity in comparison with proteasomes bound to activator PA28a $\beta$ in gel zone
II (Figure 5). It may be connected with the different content of LMP7 subunit which is known to display more pronounced ChTL activity compared to $\beta 5$ subunit. CL activity was well revealed in both gel zones.

In gel zone I corresponding to the probes of all studied brain areas of August rats, ChTL activity was higher (by $25-30 \%$ at $p<0.05$ ) and CL activity was slightly lower or showed the tendency to decrease compared to that of Wistar rats. In gel zone II corresponding to the probes of the cortex and striatum of August rats, CL activity was slightly enhanced in comparison with that of Wistar rats.
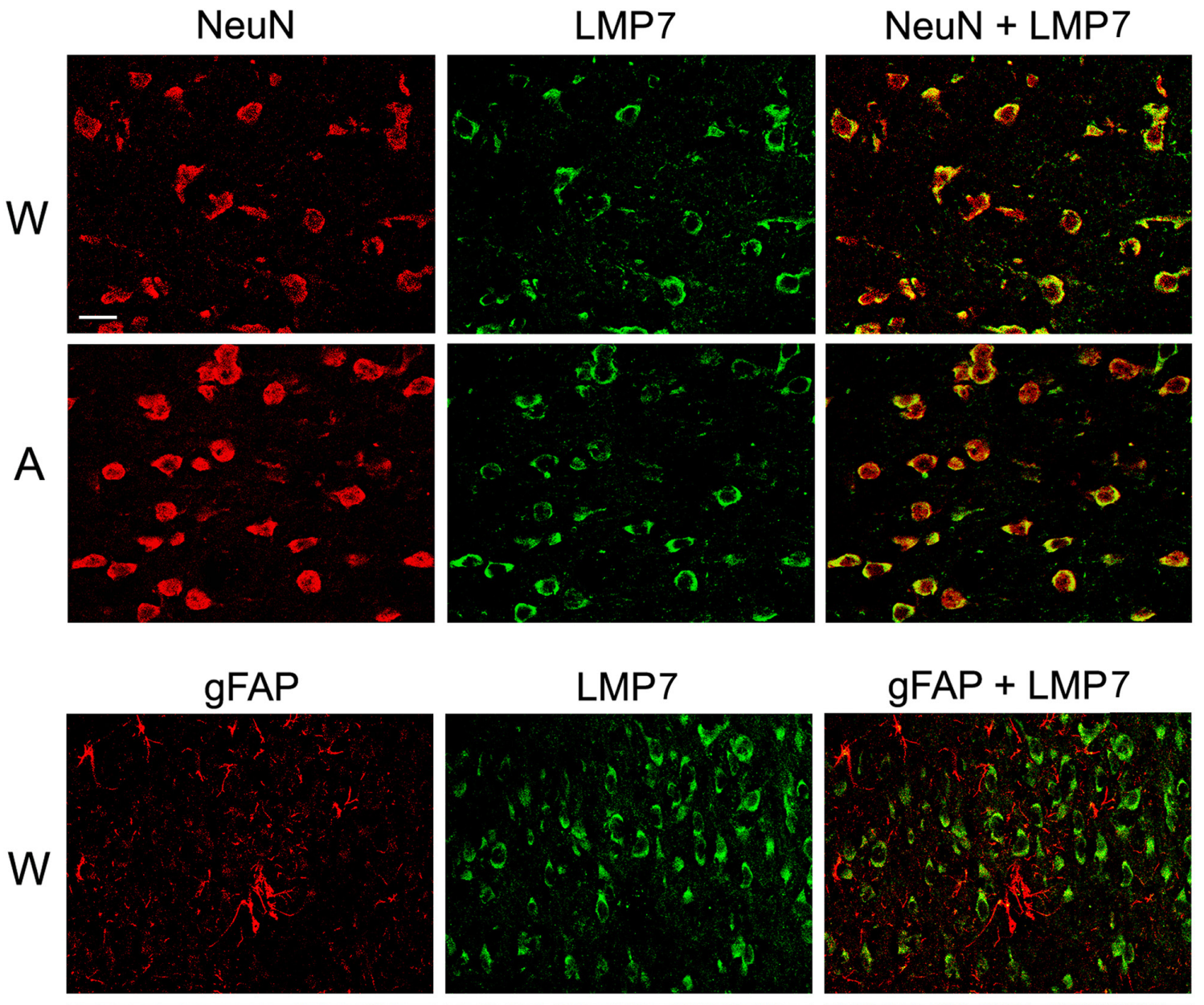

A
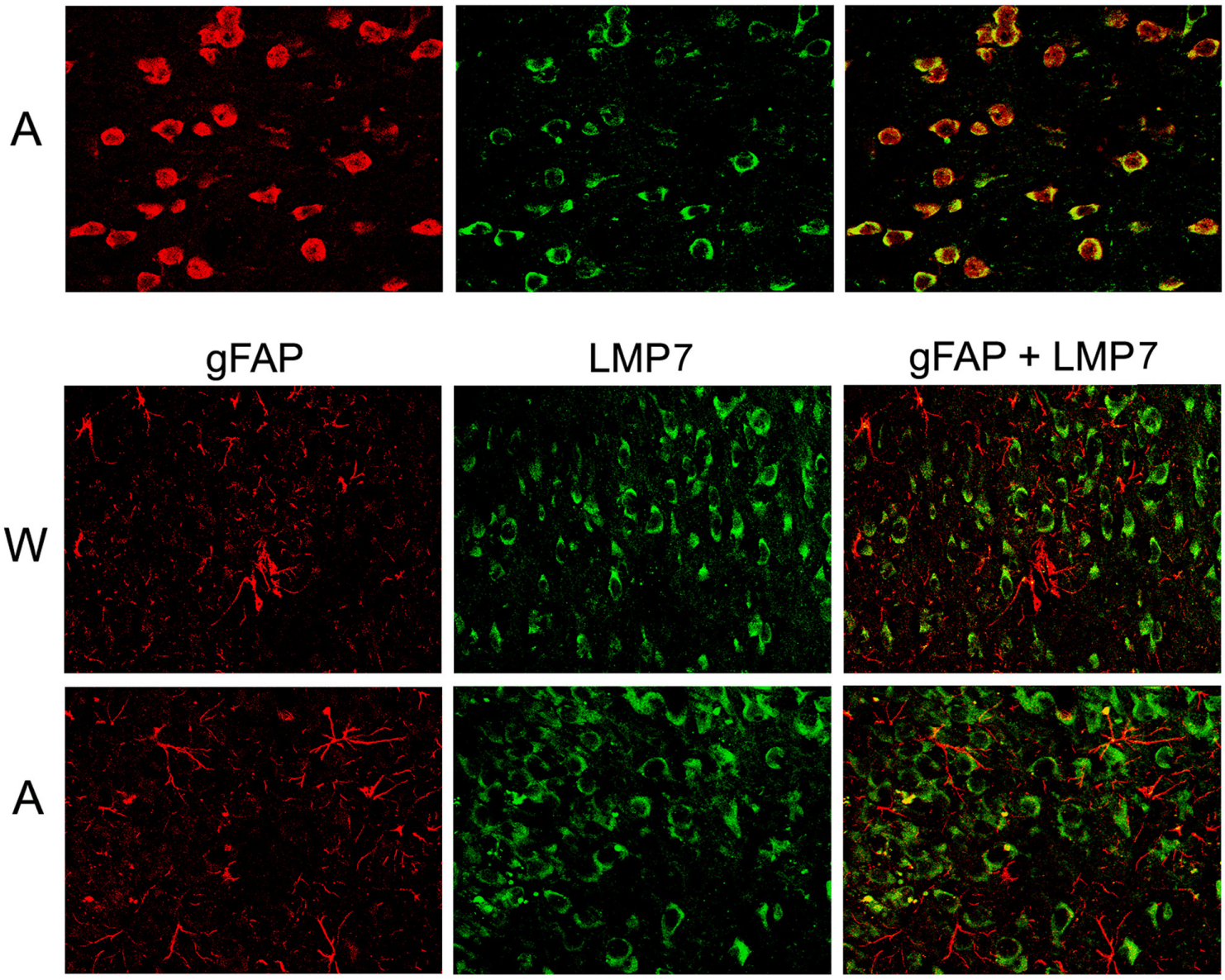

Figure 8: Distribution of proteasomes containing LMP7 subunit in brain cortex cells of Wistar and August rats. Double immunofluorescent labeling of cells in brain cortex slices was performed with the use of mouse mAb to NeuN (marker of neurons) or gFAP (marker of glial cells) and rabbit pAb to LMP7 subunit. Scale bar, $10 \mu \mathrm{m}$. W, Wistar rats; A, August rats. 
Thus, the data obtained indicate ChTL activity as the major activity for LMP7- $\beta 1$-PA700 subtype group in all studied brain areas of August rats as well as CL activity as the major activity for $\beta 5$-LMP2-PA28a $\beta$ subtype group in the cortex and striatum of August rats.

It is clear that proteasome activities, detected in a tube (Figure 7), reflected the mix of activities of multiple proteasome forms and did not reveal differences between them.

\section{Distribution and functions of the immune proteasomes in the brain}

Double immunofluorescent labeling of brain cortex cells of Wistar and August rats by antibodies to cell markers and proteasome immune subunits LMP7 and LMP2 revealed both immune subunits in neurons, but not in glial cells (Figures 8 and 9) which may play the role of antigen-presenting cells under inflammation. So, this result indicates un-immune function of the immune proteasomes in the brain cortex of Wistar and August rats.

Together with MHC class I molecules, the immune proteasomes participate in the development of synaptic plasticity. The density of MHC class I molecules on pre- and post-synaptic membranes is of great importance because it regulates neuronal activity and, possibly, represents a structural basis for long term potentiation [33, 34]. MHC class I molecules serve as reporters of neurons transmitting the information to other neurons about products of proteolysis
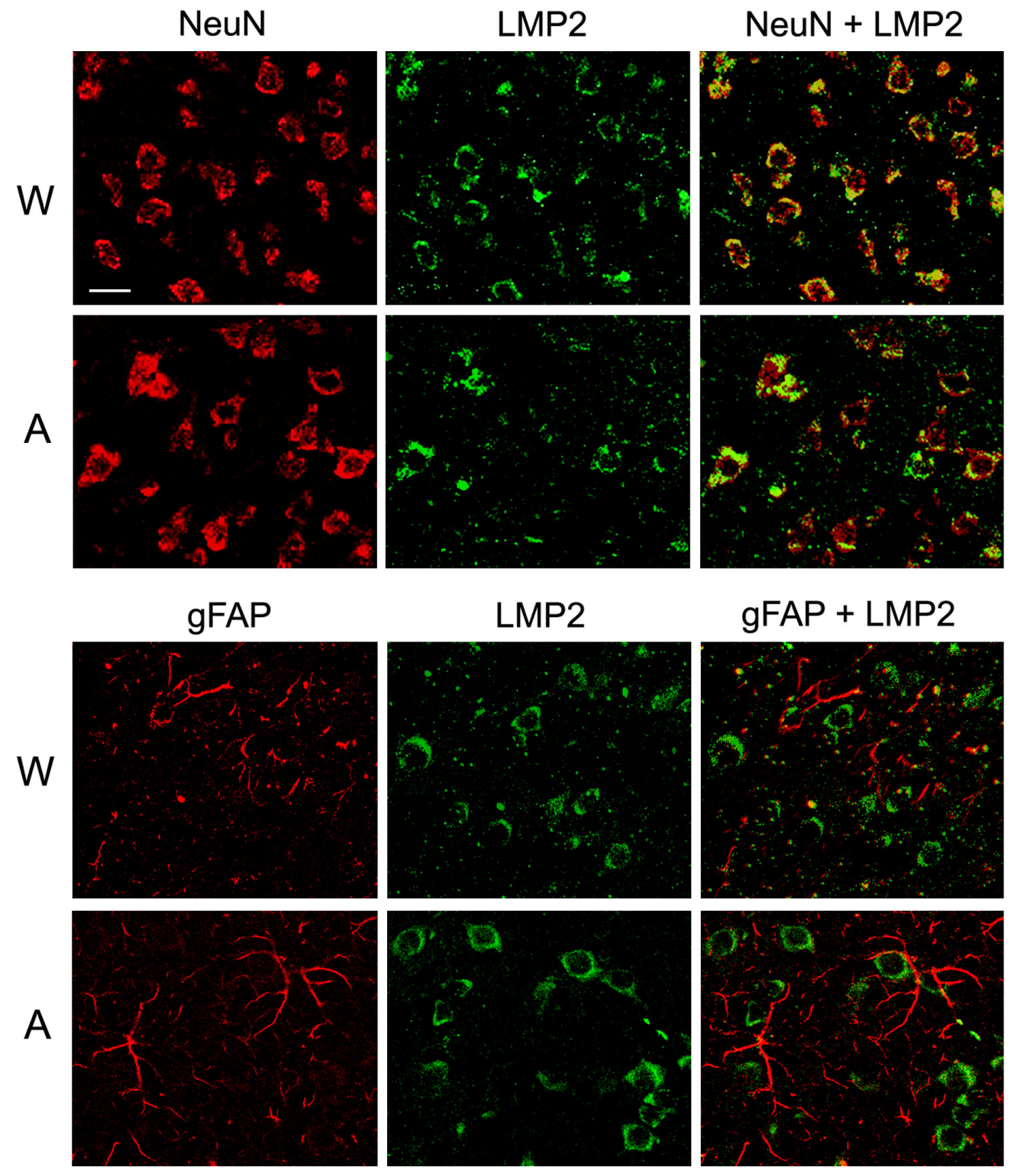

Figure 9: Distribution of proteasomes containing LMP2 subunit in brain cortex cells of Wistar and August rats. Double immunofluorescent labeling of cells in brain cortex slices was performed with the use of mouse mAb to NeuN (marker of neurons) or gFAP (marker of glial cells) and rabbit pAb to LMP2 subunit. Scale bar, $10 \mu \mathrm{m}$. W, Wistar rats; A, August rats. 
performed, predominantly, by the immune proteasomes. Besides, K. Ramachandran and S.Margolis discovered membrane proteasomes in mammalian nervous system that directly and rapidly modulated neuronal function by degrading intracellular proteins into extracellular peptides that could stimulate neuronal signaling [35].

It was earlier shown that the immune proteasomes were important for the regulation of the brain development [36] and adaptation of the brain to the stress provoked by the absence of MHC class I molecules [14]. In both these processes, the immune proteasomes is likely to generate the peptide or oligopeptide information for neuron-neuron interactions.

The pronounced immune functions of the immune proteasomes are displayed in the brain under neurodegenerative autoimmunity [37]. In oligodendrocytes of SJL mice with experimental autoimmune encephalomyelitis, the immune proteasomes containing LMP2 subunit are expressed in the enhanced quantity to generate significantly increased amounts of myelin basic protein peptides recognized by infiltrated cytotoxic lymphocytes. This leads to the loss of oligodendrocytes, axonal demyelination and development of neurodegeneration.

It would be interesting to discuss the deviations in proteasome systems of the brain areas, containing the enhanced content of dopamine and substantia nigra under Parkinson's disease accompanied by the progressive loss of dopaminergic neurons in this brain area. For Parkinson's disease, proteasome disfunction is known to be a major contributor to pathogenesis. There are decreased levels of core particle $\alpha$ subunits (the total proteasome pool) and PA700 activator, and dramatic diminishing of all three proteasome activities in the substantia nigra in sporadic Parkinson's disease brains as compared with control brains [38-41]. For August rat brain areas with the enhanced content of dopamine, we discovered the increased or unchanged level of the total proteasome pool and its retuning on the special subtypes of the immune proteasomes. So, the opposite changes in dopaminergic system are related to the radically different changes in the proteasome pool.

Thus, the present study gives the additional information about un-immune functions of the immune proteasomes in the brain. Immune subtypes LMP2PA28a $\beta$ and LMP7-PA700, detected in the enhanced quantities in August rat brain with the increased levels of monoamines, possibly produce special (oligo)peptides to provide the additional neuron-neuron interaction. Steady signaling between neurons may explain the high resistance of these rats to the acute emotional stress.

\section{MATERIALS AND METHODS}

\section{Animals}

The young males (two-month age) of inbred August rats with monoamine metabolism violations and
Wistar rats (as a control) weighing 180-200 g were used. The rats were obtained from the Department of Animal Breeding of Research Institute of General Pathology and Pathophysiology of Russian Academy of Sciences. Experiments were carried out in accordance with the European Communities Council Directive of 24 November 1986 (86/609/EEC). All efforts were made to minimize suffering. All the protocols of manipulations with animals have been approved by the Commission on Bioethics of N.K. Koltsov Institute of Developmental Biology of Russian Academy of Sciences (Permit Number: 04.17/1).

Brain sections were obtained at $8-10^{\circ} \mathrm{C}$ according to Paxinos and Watson atlas [42]: frontal cortex (Bregma: from $5.0 \mathrm{~mm}$ up to $2.76 \mathrm{~mm}$ ), striatum (Bregma: from $2.76 \mathrm{~mm}$ up to $-3.0 \mathrm{~mm}$ ), brainstem (Bregma: from -7.68 $\mathrm{mm}$ up to $-14.64 \mathrm{~mm}$ ). Brainstem area sections include parabracheal, oromotor, precerebelar, pontine reticular, facial raphe nucleus, etc.

\section{Antibodies}

We used mouse monoclonal antibodies (mAb) to proteasome $20 \mathrm{~S} \boldsymbol{\alpha} 1,2,3,5,6, \& 7$ subunits, Product Number: BML-PW8195; mouse mAb to proteasome 20S $\beta 5 \mathrm{i}$ (LMP7) subunit, Product Number: BML-PW8845; mouse $\mathrm{mAb}$ to proteasome 20S $\beta 1 \mathrm{i}$ (LMP2) subunit, Product Number: BML-PW8840; mouse mAb to proteasome $19 \mathrm{~S}$ ATPase subunit Rpt6, Product Number: BML-PW9265; mouse $\mathrm{mAb}$ to proteasome 20S $\beta 1$ subunit, Product Number: BML-PW8140; rabbit polyclonal antibodies (pAb) to proteasome 20S $\beta 5$ subunit, Product Number: BML-PW8895; rabbit pAb to proteasome 20S $\beta 5$ i (LMP7) subunit, Product Number: BML-PW8356; rabbit pAb to proteasome 20S $\beta 1$ i (LMP2) subunit, Product Number: BML-PW8205; rabbit pAb to proteasome activator $11 \mathrm{~S} \alpha$ subunit, Product Number: BML-PW8185; rabbit pAb to HSC70/HSP70, (ADI-SPA-757) (all from Enzo Life Sciences, USA); rabbit pAb to PA200, sc-135512; mouse $\mathrm{mAb}$ to $\beta$-actin, sc-81178 (from Santa Cruz Biotechnology, USA); mouse mAb to gFAP, Catalogue Number: MAB360; mouse mAb to NeuN, Catalogue Number: MAB377 (EMD Millipore, USA); goat antirabbit IgG antibodies, labeled with Alexa 488, Catalogue Number: 11035; donkey anti-mouse IgG antibodies, labeled with Alexa 594, Catalogue Number: A11034 (Invitrogen, USA).

\section{High performance liquid chromatography with electrochemical detection}

The dissected tissues of the brain parts were homogenized in $0.1 \mathrm{~N} \mathrm{HClO}_{4}$ using an ultrasonic homogenizer (L-666, MSE, England). Then $10 \mu \mathrm{l}$ of $0.2 \mathrm{~N}$ $\mathrm{HClO}_{4}$ containing $1 \mathrm{ng}$ of 2,3-dihydroxybenzoic acid was added, followed by centrifugation at 15,200 $\mathrm{g}$ for $20 \mathrm{~min}$. Supernatants were collected, frozen and stored at $-70^{\circ} \mathrm{C}$. 
High performance liquid chromatography with electrochemical detection was used to measure the content of serotonin, dopamine and their metabolites in obtained supernatants. The samples were introduced into the injector (Raininn, USA) equipped with a $20 \mu \mathrm{l}$ loop. The mobile phase consisted of $0.1 \mathrm{M}$ citrate-phosphate buffer (pH 3.2) containing $0.3 \mathrm{~mm}$ sodium octanesulfonate, 0.1 $\mathrm{mm}$ ethylenediaminetetraacetic acid, and $8 \%$ acetonitrile (all chemicals, Sigma, USA). A flow rate of $800 \mu \mathrm{l} / \mathrm{min}$ was provided by a Gilson 10SC pump (France). Peaks of serotonin, 5-hydroxyindoleacetic acid, dopamine, 3,4-dihydroxyphenylacetic acid, and homovanillic acid were identified in accordance with elution time in the standard solution, and the substance amount was estimated as a ratio of the peak area of the internal standard solution to that of the specimen by using a software support (Multichrom 1.5, Ampersand Ltd.).

\section{Preparation of brain extracts}

Extracts of the brain cortex, striatum and stem were prepared at $4{ }^{\circ} \mathrm{C}$. Tissues were mechanically homogenized in three volumes (w/v) of buffer containing $50 \mathrm{mM} \mathrm{Na}$ HEPES, $\mathrm{pH} 7.5,200 \mathrm{mM} \mathrm{NaCl}$, and $10 \mathrm{mM}$ EDTA. Homogenates were centrifuged under $18,000 \mathrm{~g}$ for 1 h. Obtained supernatants (extracts) were employed as samples for SDS PAGE, electrophoresis in native conditions and detection of proteasome activities.

\section{Electrophoresis in native conditions}

All procedures were performed at $4{ }^{\circ} \mathrm{C}$. After the addition of sucrose (to $10 \%$ ), extracts were subjected to the electrophoresis ( $10 \mu \mathrm{l}, 40 \mu \mathrm{g}$ of protein per lane) in a starting $3 \%$ polyacrylamide gel, prepared in $50 \mathrm{mM}$ BisTris / Glycine buffer, pH 8.0 containing $1 \mathrm{mM}$ EDTA, and then in a $4-10 \%$ polyacrylamide gradient gel (acrylamide : bis-acrylamide, $19: 1)$, prepared in the same buffer. Electrophoresis was performed at $60 \mathrm{~V}$ for $14 \mathrm{~h}, 140 \mathrm{~V}$ for $10 \mathrm{~h}$ and finally at 260 $\mathrm{V}$ for $20 \mathrm{~h}$ with utilizing the same buffer.

To visualize proteasome proteolytic activities, $200 \mathrm{mM}$ Na-HEPES buffer, $\mathrm{pH} 7.5$, containing $300 \mu \mathrm{M}$ fluorogenic substrate N-succinyl-leu-leu-val-tyr-amido4-methyl coumarin (Suc-LLVY-AMC) or Z-Leu-LeuGlu-amido-4-methyl coumarin (Z-LLE-AMC) (1/20 of gradient gel volume) was placed to gel surface. The gel was covered by cellulose acetate film and incubated at $37^{\circ} \mathrm{C}$ for $20 \mathrm{~min}$. Fluorescence bands in the gel were photographed in a dark room under illumination at 365 $\mathrm{nm}$. Then the gel was incubated in the solution containing $0.025 \mathrm{M}$ Tris, $0.19 \mathrm{M}$ glycine, $\mathrm{pH} 8.3$, and $10 \mathrm{M}$ carbamide at the room temperature for $30 \mathrm{~min}$ under gentle agitation. This solution was also applied for the following semidry transfer of polypeptides onto Hybond-ECL membrane (Amersham). The use of carbamide instead of SDS for disruption of large protein complexes provides much better polypeptide transfer and allows us to obtain the reproducible results.

\section{Western blot analysis}

After SDS electrophoresis in 13\% polyacrylamide gel (15 $\mu \mathrm{l}$ of extract, $60 \mu \mathrm{g}$ of protein per lane), polypeptides were transferred from the gel onto Hybond-ECL membrane (Amersham) by the standard procedure. After SDS and native PAGE, immunodetection was carried out with the use of primary antibodies to subunits a1,2,3,5,6,7, LMP7 (mAb), LMP2 (mAb) (1 : 1000), $\beta 1, \beta 5$, PA28a, Rpt6 (1 $: 1500)$, and proteins PA200, HSP / HSC70s, (1:1500), $\beta$ actin $(1: 1000)$ and corresponding secondary antibodies peroxidase conjugated. The image analysis was performed using standard "ImageJ" software. The relative quantities (optical density) of the immunoreactive bands on X-ray film were measured. The dependence of the optical density on the amount of the protein subjected to Western blotting was evaluated preliminarily. For further processing, portions were taken within a proportional dose range. Results were normalized to $\beta$ actin content detected by Western blotting after SDS PAGE.

\section{Determination of proteasome activities}

Proteasome ChTL activity and CL activity were determined by hydrolysis of fluorogenic substrates SucLLVY-AMC (Sigma, USA) and Z-LLE-AMC (Tebu-Bio, Belgium), respectively. The activity was determined in portions of $0.5-2 \mu \mathrm{l}$ of brain extracts in final volume of 100 $\mu \mathrm{l}$ of reaction mix containing $50 \mathrm{mM} \mathrm{Na}-\mathrm{HEPES},(\mathrm{pH} 7.5$ ), $1 \mathrm{mM}$ DTT, and $30 \mu \mathrm{M}$ Suc-LLVY-AMC or Z-LLE-AMC.

In Supplementary Figure 1, the time dynamics of proteasome ChTL and CL activities in brain extracts of Wistar and August rats obtained with the use of DTX 880 Beckman Coulter and Multimode Analysis Software is presented. On the basis of the results showing the linear dependence of proteasome activities on reaction time, interval of 20 min was chosen for experiments.

So, the reactions were carried out at $37^{\circ} \mathrm{C}$ for 20 min and terminated by the addition of $1 \%$ SDS. The digestion product was detected by using a fluorimeter with the excitation wavelength of $380 \mathrm{~nm}$ and the emission wavelength of $440 \mathrm{~nm}$. Proteasome-independent activity was determined in the presence of $5 \mu \mathrm{M}$ of inhibitor of proteasome activities, Z-leucyl-leucyl-leucinal (MG-132) (Sigma, USA) (less than 10\% activity in all samples) and subtracted from the values obtained in the absence of MG132. In final, proteasome activities were normalized to 1 $\mathrm{mg}$ of protein, detected by Lowry method [43].

\section{Immunohistochemistry}

Rats were anesthetized with pentobarbital $(97.2 \mathrm{mg} /$ kg i.p.), briefly perfused transcardially with saline and 
then with $4 \%$ paraformaldehyde in phosphate buffer $(0.1$ $\mathrm{M}, \mathrm{pH}$ 7.4) for $5 \mathrm{~min}$. Brains were dissected, post-fixed in buffered paraformaldehyde for $2 \mathrm{~h}$ at room temperature, equilibrated with $25 \%$ sucrose in phosphate buffer at $4{ }^{\circ} \mathrm{C}$, frozen, and cut into coronal $12-\mu \mathrm{m}$ sections on slides using a cryostat Leica CM 1900. Brain structures of Wistar and August rats were mounted on the same slides.

For double staining, frontal cortex slices were blocked with 7\% BSA and $0.3 \%$ Triton X100 in PBS for $1 \mathrm{~h}$, and then subjected to antibody treatment. Antigen localization was determined by incubation of the slices with $\mathrm{pAb}$ to LMP2 or LMP7 (1 : 500) with 5\% BSA and 0.1\% Triton $\mathrm{X} 100$ in PBS overnight at $4^{\circ} \mathrm{C}$. After incubation with the primary antibodies, slices were washed four times $(5 \mathrm{~min}$ per wash) with PBS and treated with the goat secondary anti-rabbit IgG antibodies, Alexa 488-conjugated (1:800) for $2 \mathrm{~h}$. After four washes with PBS (10 min per wash), the slides were incubated with other primary $\mathrm{mAb}$ to NeuN or gFAP $(1: 1000)$ with $5 \%$ BSA and $0.1 \%$ Triton X100 in PBS overnight at $4{ }^{\circ} \mathrm{C}$, washed four times ( 5 min per wash) with PBS and then treated with the donkey secondary antimouse IgG antibodies, Alexa 594-conjugated $(1: 700)$ for $2 \mathrm{~h}$. After four washes with PBS (10 min per wash) slides were mounted with the Mowiol and analyzed under confocal microscope Leica SPE equipped with an Ar-Kr laser at the Core Facility on Cell Technologies and Optical Research Methods in Developmental Biology of N.K. Koltzov Institute of Developmental Biology of Russian Academy of Sciences. To ensure equal illumination for all treatments, the same intensity and filter settings were used throughout. Images were recorded at a resolution of $1024 \times 1024$ pixels and processed with the Leica LCS software. Control experiments were performed by omitting primary or secondary antibodies.

\section{Statistics}

Results are presented as mean value of data measured for at least three parallel samples in one experiment and obtained in 6-8 experiments. Differences relative to control values were assessed using KruskalWallis test of ANOVA and 95\% confidence interval of the mean. Significant differences were considered to be at $p<0.05$. The data were treated with F-test to compare variances. The statistical analysis program Statistica 8.0 (Statsoft, 2008) was used.

\section{Abbreviations}

HSP, heat shock protein; MHC, major histocompatibility complex; CL, caspase-like; ChTL, chymotrypsin-like; mAb, monoclonal antibodies; $\mathrm{pAb}$, polyclonal antibodies; Suc-LLVY-AMC, N-succinylleu-leu-val-tyr-amido-4-methyl coumarin; Z-LLE-AMC, Z-Leu-Leu-Glu-amido-4-methyl coumarin; MG-132, Z-leucyl-leucyl-leucinal.

\section{Author contributions}

NS, PE and YL designed the study. PE, YL, AR, AK and $\mathrm{YN}$ collected the data. NS, PE and YL analyzed the data. NS and PE interpreted the data. NS and YL prepared illustrations and wrote the manuscript. NS and PE edited the manuscript. All authors critically reviewed the content and approved the final version before the submission.

\section{CONFLICTS OF INTEREST}

There is no any conflicts of interest.

\section{FUNDING}

This work was supported by the Russian Foundation for Basic Research (grant number 15-04-03494) and government program of basic research (number 01082016-0003).

\section{REFERENCES}

1. Lapin IP, Oxenkrug GF. Intensification of the central serotoninergic processes as a possible determinant of the thymoleptic effect. Lancet. 1969; 1:132-136.

2. Wise CD, Berger BD, Stein L. Benzodiazepines: anxietyreducing activity by reduction of serotonin turnover in the brain. Science. 1972; 177:180-183.

3. Graeff FG. On serotonin and experimental anxiety. Psychopharmacology. 2002; 163:467-476.

4. Lauder JM. Neurotransmitters as growth regulatory signals: role of receptors and second messengers. Trends Neurosci. $1993 ; 16: 233-240$.

5. Gorbunova AV. Action of immobilization stress on biogenic amines in the brain: the role of genotype. Neurochem. 2006; 23:42-46.

6. Khudoerkov RM, Dovedova EL, Khrustalev DA. [The influence of amphetamine on changes in the brain neuromediator metabolism]. [Article in Russian]. Zh Nevrol Psikhiatr Im S S Korsakova. 2007; 107:49-54.

7. Dovedova EL, Khrustalev DA. Comparative characteristics of the enzyme systems of exchange of biogenic amines in the brain of rats Wistar and August under various doses of amphetamine in vivo. Neurochem. 2007; 24:150-155.

8. Malysheva EV, Kruglov SV, Khomenko IP, Bakhtina LY, Pshennikova MG, Manukhina EB, Malyshev IY. Role of extracellular and intracellular nitric oxide in the regulation of macrophage responses. Bull Exp Biol Med. 2006; 141:404-406.

9. Pshennikova MG, Popkova EV, Pokidyshev DA, Khomenko IP, Zelenina OM, Kruglov SV, Manukhina EB, Shimkovich MV, Goriacheva AV, Malyshev IuI. [The effects of adaptation to hypoxia on the resistance to 
neurodegenerative disorders in the brain of rats of different genetic strains]. [Article in Russian]. Vestn Ross Akad Med Nauk. 2007; 2:50-55.

10. Goryacheva AV, Kruglov SV, Pshennikova MG, Smirin BV, Malyshev IY, Barskov IV, Viktorov IV, Downey HF, Manukhina EB. Adaptation to intermittent hypoxia restricts nitric oxide overproduction and prevents beta-amyloid toxicity in rat brain. Nitric Oxide. 2010; 23:289-299.

11. Grune T, Reinheckel T, Davies KJ. Degradation of oxidized proteins in K562 human hematopoietic cells by proteasome. J Biol Chem. 1996; 271:15504-15509.

12. Raynes R, Pomatto LC, Davies KJ. Degradation of oxidized proteins by the proteasome: distinguishing between the 20S, 26S, and immunoproteasome proteolytic pathways. Mol Aspects Med. 2016; 50:41-55.

13. Kotamraju S, Matalon S, Matsunaga $T$, Shang $T$, Hickman-Davis JM, Kalyanaraman B. Upregulation of immunoproteasomes by nitric oxide: potential antioxidative mechanism in endothelial cells. Free Rad Biol Med. 2006; 40:1034-1044.

14. Lyupina YV, Bogatyrev ME, Orlova AS, Marjukhnich EV, Kazansky DB, Sharova NP. Proteasomes in the brain of $\beta 2$-microglobulin knockout mice. Biochemistry (Mosc). 2013; 78:1124-1133.

15. Tanaka K. The proteasome: overview of structure and functions. Proc Jpn Acad Ser B Phys Biol Sci. 2009; 85:12-36.

16. Groettrup M, Standera S, Stohwasser R, Kloetzel PM. The subunits MECL-1 and LMP2 are mutually required for incorporation into the $20 \mathrm{~S}$ proteasome. Proc Natl Acad Sci U S A. 1997; 94:8970-8975.

17. Griffin TA, Nandi D, Cruz M, Fehling HJ, van Kaer L, Monaco JJ, Colbert RA. Immunoproteasome assembly. Cooperative incorporation of interferon c (IFNc)-inducible subunits. J Exp Med. 1998; 187:97-104.

18. Tanaka K, Kasahara M. The MHC class I ligand-generating system: roles of immunoproteasomes and the interferongamma-inducible proteasome activator PA28. Immunol Rev. 1998; 163:161-176.

19. Unno M, Mizushima T, Morimoto Yu, Tomisugi Yo, Tanaka $\mathrm{K}$, Yasuoka N, Tsukihara T. The structure of the mammalian $20 \mathrm{~S}$ proteasome at $2.75 \mathrm{E}$ resolution. Structure. 2002; 10:609-618.

20. Voigt A, Salzmann U, Seifert U, Dathe M, Soza A, Kloetzel PM, Kuckelkorn U. 20S proteasome dependent generation of an IEpp89 murine cytomegalovirus derived H 2L(d) epitope from a recombinant protein. Biochem Biophys Res Commun. 2007; 355:549-554.

21. Yuksek K, Chen WL, Chien D, Ou JH. Ubiquitin independent degradation of hepatitis $\mathrm{C}$ virus $\mathrm{F}$ protein. $\mathrm{J}$ Virol. 2009; 83:612-621.

22. Fonseca PC, He J, Morris EP. Molecular model of the human 26S proteasome. Mol Cell. 2012; 46:54-66.
23. Tomko RJ Jr, Hochstrasser M. Molecular architecture and assembly of the eukaryotic proteasome. Annu Rev Biochem. 2013; 82:415-445.

24. Kloetzel PM. Antigen processing by the proteasome. Nat Rev Mol Cell Biol. 2001; 2:179-187.

25. Rock KL, York IA, Saric T, Goldberg AL. Protein degradation and the generation of MHC class I-presented peptides. Adv Immunol. 2002; 80:1-70.

26. Ortega J, Heymann JB, Kajava AV, Ustrell V, Rechsteiner $\mathrm{M}$, Steven AC. The axial channel of the $20 \mathrm{~S}$ proteasome opens upon binding of the PA200 activator. J Mol Biol. 2005; 346:1221-1227.

27. Frentzel S, Kuhn-Hartmann I, Gernold M, Gott P, Seelig A, Kloetzel PM. The major-histocompatibility-complexencoded beta-type proteasome subunits LMP2 and LMP7. Evidence that LMP2 and LMP7 are synthesized as proproteins and that cellular levels of both mRNA and LMP-containing 20S proteasomes are differentially regulated. Eur J Biochem. 1993; 216:119-126.

28. Lyupina YV, Abaturova SB, Erokhov PA, Orlova OV, Beljelarskaya SN, Mikhailov VS. Proteotoxic stress induced by Autographa californica nucleopolyhedrovirus infection of Spodoptera frugiperda Sf9 cells. Virology. 2013; 436:49-58.

29. Tai HC, Besche H, Goldberg AL, Schuman EM. Characterization of the brain $26 \mathrm{~S}$ proteasome and its interacting proteins. Front Mol Neurosci. 2010; 3. https:// doi.org/10.3389/fnmol.2010.00012.

30. Shibatani T, Carlson EJ, Larabee F, McCormack AL, Fruh K, Skach WR. Global organization and function of mammalian cytosolic proteasome pools: implications for PA28 and 19S regulatory complexes. Mol Biol Cell. 2006; 17:4962-4971.

31. Stepanova AA, Lyupina YV, Sharova NP, Erokhov PA. Native structure of rat liver immune proteasomes. Dokl Biochem Biophys. 2016; 468:200-202.

32. Guillaume B, Chapiro J, Stroobant V, Colau D, Van Holle B, Parvizi G, Bousquet-Dubouch MP, Théate I, Parmentier $\mathrm{N}$, Van den Eynde BJ. Two abundant proteasome subtypes that uniquely process some antigens presented by HLA class I molecules. Proc Natl Acad Sci U S A. 2010; 107:18599-18604.

33. Boulanger LM, Shatz CJ. Immune signalling in neural development, synaptic plasticity and disease. Nat Rev Neurosci. 2004; 5:521-531.

34. Shatz CJ. MHC class I: an unexpected role in neuronal plasticity. Neuron. 2009; 64:40-45.

35. Ramachandran KV, Margolis SS. A mammalian nervoussystem-specific plasma membrane proteasome complex that modulates neuronal function. Nat Struct Mol Biol. 2017; 24:419-430.

36. Orlova AS, Liupina YV, Abaturova SB, Sharova NP. Distinctive features of immune proteasome expression 
during the development of the central nervous system in rats. Rus J Bioorg Chem. 2014; 40:649-657.

37. Belogurov A Jr, Kuzina E, Kudriaeva A, Kononikhin A, Kovalchuk S, Surina Y, Smirnov I, Lomakin Y, Bacheva A, Stepanov A, Karpova Y, Lyupina Y, Kharybin O, et al. Ubiquitin-independent proteosomal degradation of myelin basic protein contributes to development of neurodegenerative autoimmunity. FASEB J. 2015; 29:1901-1913.

38. McNaught KS, Jenner P. Proteasomal function is impaired in substantia nigra in Parkinson's disease. Neurosci Lett. 2001; 297:191-194.

39. McNaught KS, Belizaire R, Isacson O, Jenner P, Olanow CW. Altered proteasomal function in sporadic Parkinson's disease. Exp Neurol. 2003; 179:38-46.
40. Tofaris GK, Razzaq A, Ghetti B, Lilley KS, Spillantini MG. Ubiquitination of alpha-synuclein in Lewy bodies is a pathological event not associated with impairment of proteasome function. J Biol Chem. 2003; 278:44405-44411.

41. Snyder H, Wolozin B. Pathological proteins in Parkinson's disease: focus on the proteasome. J Mol Neurosci. 2004; 24:425-442.

42. Paxinos $\mathrm{G}$, Watson $\mathrm{C}$. The rat brain in stereotaxic coordinates. 5th ed. Burlington, MA: Elsevier Academic Press; 2005.

43. Lowry OH, Rosebrough NJ, Farr AL, Randall RJ. Protein measurement with the Folin phenol reagent. J Biol Chem. $1951 ; 193: 265-275$. 